\title{
WIND TURBINE GENERATOR SLIP RING DAMAGE DETECTION THROUGH TEMPERATURE DATA ANALYSIS
}

\author{
Davide ASTOLFI, Francesco CASTELLANI, Francesco NATILI
}

University of Perugia, Department of Engineering, Via Duranti, 06125, Perugia, Italy, davide.astolfi@unipg.it, francesco.castellani@unipg.it, francesco.natili@yahoo.it

\begin{abstract}
The use of condition monitoring techniques in wind energy has been recently growing and the average unavailability time of an operating wind turbine in an industrial wind farm is estimated to be less than the $3 \%$. The most powerful approach for gearbox condition monitoring is vibration analysis, but it should be noticed as well that the collected data are complex to analyse and interpret and that the measurement equipment is costly. For these reasons, several wind turbine subcomponents are monitored through temperature sensors. It is therefore valuable developing analysis techniques for this kind of data, with the aim of detecting incoming faults as early as possible. On these grounds, the present work is devoted to a test case study of wind turbine generator slip ring damage detection. A principal component regression is adopted, targeting the temperature collected at the slip ring. Using also the data collected at the nearby wind turbines in the farm, it is possible to identify the incoming fault approximately one day before it occurs.
\end{abstract}

Keywords: wind energy, wind turbines, fault diagnosis, condition monitoring, principal component regression

\section{INTRODUCTION}

In the latest years, wind turbines technology has been characterized by a considerable efficiency improvement: a turning point has been the optimization of the control system. It is remarkable that this can be conceived at the level of single wind turbine (through yaw [1] or pitch [2,3] or cutout $[4,5]$ optimization) or at the wind farm level (through cooperative control $[6,7]$ or wind turbine wakes steering [8]).

Another fundamental direction for wind turbine production improvement is diminishing the unavailability time. According to a recent estimate [9], a wind turbine operating in an industrial wind farm has unavailability time less than $3 \%$ and therefore, in perspective, target $100 \%$ availability is becoming more and more realistic.

Optimizing wind turbine availability means preventing faults sufficiently early to avoid production losses. For this reason, wind turbine condition monitoring systems, based on vibration measurement at meaningful parts of the gearbox, are becoming more and more widespread. The target of this kind of equipment is typically the gearbox $[10,11]$ because repairing gearboxes is particularly costly.

Nevertheless, there are several other wind turbine subcomponents that are worth being monitored [12]: for example, yaw and pitch motors, generator bearings and slip rings, and so on.

This kind of subcomponents is typically monitored through temperature sensors [13-16], because they are cheaper with respect to accelerometers and because the data are relatively easier to interpret with respect to vibration data. It should be noticed that the analysis of operation data, collected through Supervisory Control And Data Acquisition (SCADA) systems, is not as powerful as vibration analysis, as regards the diagnosis time. Exactly for this reason, it is particularly important to empower the SCADAbased condition monitoring [17-20] through advanced analysis techniques [21-25].

On these grounds, the present work is devoted to a test case discussion: the selected test case is a fault at the generator slip ring of a $2 \mathrm{MW}$ wind turbine from a wind farm sited in Italy, featuring in total nine 2 MW aerogenerators. In general, damages occurring at wind turbine generators are particularly elusive to diagnose [26-28] and most likely it happens that the fault is identified too late for timely intervention. For this reason, particular attention has been devoted to the formulation of an appropriate method for the fault diagnosis. The idea is modelling the temperature collected at the slip ring temperature as a linear function of operating parameters of the wind turbine and, more importantly, of the other available internal temperature measurements. The selected model is principal component regression and the objective is identifying the occurring fault by detecting peculiar characteristics of the residuals between slip ring temperature measurements and model estimates.

The main result of this study is that it is possible diagnosing the generator slip ring fault for the test case of interest approximately one day before it occurs: on the grounds of the above discussion 
about the features of generator faults, this diagnosis time is remarkable.

The structure of the manuscript is as follows: in Section 1, the wind farm and the data set are described. In Section 2, the method is presented. The results are collected and discussed in Section 3 and, finally, conclusions are drawn and further directions of this work are indicated in Section 4.

\section{THE WIND FARM AND THE DATA SET}

The test case of the present work is a wind farm sited in southern Italy, whose layout is sketched in Figure 1. The main features of the wind turbines are presented in Table 1. WTG09 is indicated in red because it is the wind turbine that has undergone a generator slip ring damage.

Table 1: Main turbine characteristics

\begin{tabular}{|l|l|}
\hline Number of turbines & 9 \\
\hline Rotor diameter & 100 meters \\
\hline Hub height & 95 meters \\
\hline Rated power & $2 \mathrm{MW}$ \\
\hline Terrain & Flat \\
\hline
\end{tabular}

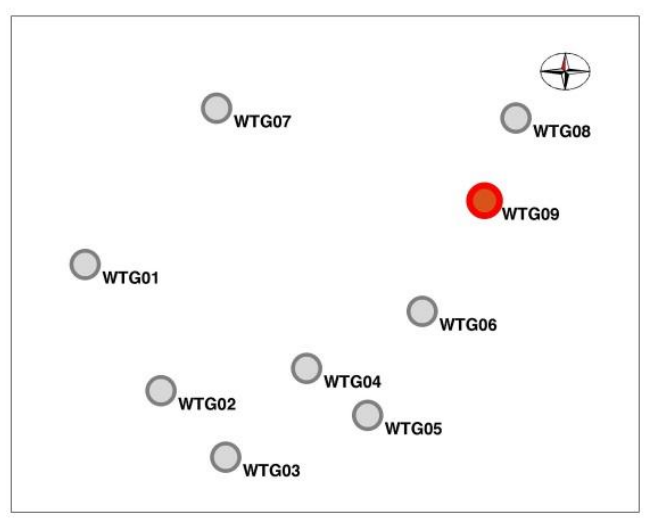

Fig. 1. The layout of the wind farm

The measurements at disposal for the study are:

- nacelle wind speed and direction;

- power production;

- ambient temperature;

- yaw error;

- rotor revolutions per minute;

- generator revolutions per minute;

- reference blade pitch angle;

- generator bearing temperature;

- gear oil temperature;

- gearbox main bearing temperatures (collected at three different points);

- generator slip ring temperature.

The data set at disposal goes from 01st September 2018 to 11th January 2019 (the day of fault occurrence at WTG09). The data have been filtered on the request that each wind turbine in the farm was productive: the appropriate run time counter, available in the SCADA data set, has been used for this purpose. Upon filtering, the data sets results being composed of 16826 data for each of the measurement channels.

\section{THE METHOD}

The idea of the present work is detecting the generator slip ring fault occurrence through temperature data analysis.

In Figure 2, a time series plot for WTG09 is reported: in y-axis, there is the generator slip ring temperature (relative to the ambient temperature), and in $\mathrm{x}$-axis there is the time. The latest days before the fault occurrence are included in the plot. From Figure 2, it qualitatively arises that maximum observed temperature increases approaching the fault occurrence.

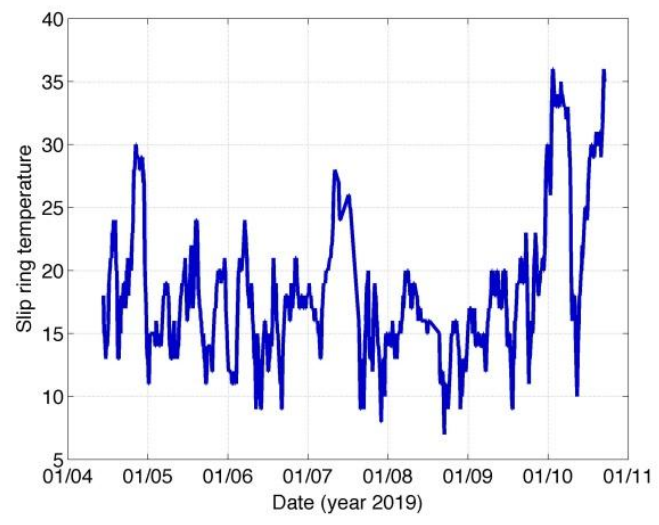

Fig. 2. Generator slip ring temperature time series: WTG09, one week before fault occurrence

On these grounds, the objective of the present work is formulating a reliable model for the generator slip ring temperature as a function possibly of the ambient conditions, operation parameters of the wind turbine and thermal conditions at wind turbine subcomponents. Once the model is trained with data describing the normal functioning of the wind turbine and of the subcomponent of interest (the slip ring generator, in this case), hopefully the fault occurrence can be detected in the validation data set as anomalous features of the residuals between model estimates and measurements. It should be noticed that this approach is general and doesn't depend on the subcomponent of interest for the particular test case study.

The target of the model has therefore been selected as the generator slip ring temperature (relative to the ambient temperature) and the possible input variables for the model are all the other measurements listed in Section 1. As discussed for example in [30-32], a linear model is commonly adequate for this kind of tasks. A subtle point regards the fact that there likely is a remarkable collinearity between the possible input variables of the model (for example, wind speed and power production) and this can affect the quality of the model. For this reason, the principal component regression has been selected for this 
study. Sideways, it should be noticed that the use principal component analysis has been recently spreading for wind energy applications [33-35] and engineering applications in general [36-38].

The method goes as follows. Let $\mathrm{y}_{\mathrm{n}, 1}$ be the vector of measured output (namely, the slip ring temperature relative to the ambient temperature) and $X_{n, o}$ be the matrix of $p$ covariatives. The standard least squares regression poses that

$$
y=X \beta+\varepsilon
$$

where $\beta$ is the vector of the regression coefficients that must be estimated from the data and $\varepsilon$ is a vector of random errors. The ordinary least squares estimate of $\beta$ is

$$
\beta_{\mathrm{ols}}=\left(\mathrm{X}^{\mathrm{T}} \mathrm{X}\right)^{-1} \mathrm{X}^{\mathrm{T}} \mathrm{Y}
$$

The principal component estimate of $\beta$ is obtained from the singular value decomposition of $X$. Let

$$
\mathrm{X}=\mathrm{U} \Delta \mathrm{V}^{\mathrm{T}}
$$

where the columns of $U$ and $V$ are orthonormal sets of vectors denoting respectively the left and right singular vectors of $X$ and $\Delta$ is a diagonal matrix. This allows decomposing $X X^{T}$ as

$$
\mathrm{XX}^{\mathrm{T}}=\mathrm{V} \Lambda \mathrm{V}^{\mathrm{T}}
$$

where $\Lambda=\operatorname{diag}\left(\lambda_{1}, \ldots, \lambda_{p}\right)$ and $\lambda_{1} \geq \cdots \geq \lambda_{p} \geq$ $0 . X V_{i}$ is the $\mathrm{i}$-th principal component and $V_{i}$ is the $\mathrm{i}$-th loading corresponding to the $\mathrm{i}$-th principal value $\lambda_{i}$. Therefore, the transformed matrix $W=X V$ can be viewed as a new covariatives matrix and the principal component regression is basically an ordinary least squares regression between y and $W$.

The principal component regression can be superior with respect to ordinary least squares when the covariatives are severely collinear and this can be seen from Equation (4). Namely, the $W$ matrix can be truncated up to a desired number of principal components. This procedure means neglecting the smallest eigenvalues in the decomposition (4) and this exactly means addressing collinearity, because, when two covariatives are highly collinear, $X$ tends losing its full rank and $X X^{T}$ has eigenvalues tending to 0 .

Finally, the principal component estimate of $\beta$ is given by

$$
\beta_{\mathrm{pcr}}=\mathrm{V}\left(\mathrm{W}^{\mathrm{T}} \mathrm{W}\right)^{-1} \mathrm{~W}^{\mathrm{T}} \mathrm{Y}
$$

The idea is employing the data of a wind turbine having normal functioning for constructing the baseline model (namely, computing $\beta_{p c r}$ ). Subsequently, the model will be employed on two test data sets: one from a healthy wind turbine and one from WTG09. The slip ring temperature on the test data sets will be simulated through the following formula:

$$
\mathrm{Y}_{\text {test }}=\mathrm{X}_{\text {test }} \beta_{\mathrm{pcr}}
$$

and the target to analyze will be the difference in the behavior of the residuals between model estimates and measurements. In the present work, results are reported for the particular selection of
WTG04 as training wind turbine and of WTGs 06 and 09 as test wind turbines. It should be noticed that results are reported for this particular selection of training and test wind turbines only for brevity. In practice, each possible selection of healthy couples of wind turbines has been tested for training and validating the model and it has been observed that the results do not depend on the particular couple selected. Therefore, it has been observed that the results are as general as possible, compatibly with the type and kind of data sets at disposal.

The training healthy wind turbine (WTG04, in this case) data set is employed for estimating an adequate number of principal components for the model. Recall that the total number of covariatives at disposal for modelling the output is 13 (Section 1). K-fold cross validation [39] of the model, for different numbers of principal components, is performed. The procedure goes as follows: $(\mathrm{K}-1) / \mathrm{K}$ of the test healthy wind turbine (WTG06) data are used for training and the remaining $1 / \mathrm{K}$ are used for testing. As a rule of thumb, $\mathrm{K}$ is commonly selected to be 10 and this value is adopted in this study. The $(\mathrm{K}-1) / \mathrm{K}$ fraction of the data is used for estimating $\beta$ through Equation 5 and the $1 / \mathrm{K}$ fraction is used for testing through Equation 6. For each fold selection, the Root Mean Square Error (RMSE) is selected as metric for quantifying the goodness of the regression:

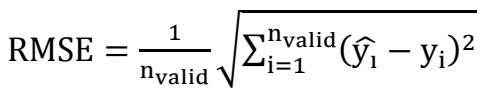

where $\widehat{y}_{i}$ is the model estimate and $y_{i}$ is the measurement of the slip ring temperature. The $R M S E$ values are subsequently averaged on the folds selection and therefore, for a given number of principal components included in the model, one has a unique metric for quantifying the goodness of the regression. The results are reported in Figure 3 and it arises that 10 principal components are needed for obtaining a RMSE of the order of 2 degrees.

\section{THE RESULTS}

In Figure 4 a time series plot is reported. The absolute difference between the slip ring temperature measurements $\boldsymbol{y}_{\boldsymbol{i}}$ and the model estimates $\widehat{y}_{\boldsymbol{\imath}}$ is reported for the two test wind turbines (the healthy reference one WTG06 and the faulty one WTG09). The data set covers the week before the fault occurrence and the consequent WTG09 shutdown. From Figure 4, it arises that the fault occurrence becomes clearly visible 16 hours before WTG09 shuts down: the absolute difference between measurements and model estimates doubles with respect to the order of magnitude of its peak when the wind turbine is in normal functioning. It should be noticed that this analysis 
has been done for all the length of the data set at disposal and the conclusions from Figure 4 are confirmed. Figure 4 displays one week (the nearest to the fault occurrence) of residuals only for plot readability. A quantitative criterion for diagnostic threshold selection has been formulated as well. The mean and the standard deviation of the absolute residuals for the healthy test wind turbine (WTG06, in this case) are computed. It can be assumed that these residuals have a Gaussian distribution with a fair approximation and, therefore, a deviation with respect to the average larger that 3 standard deviations corresponds to a $99.7 \%$ probability rule. This diagnostic threshold is reported in Figure 4 as a black dashed line and it arises that, with this rule, the damage is detected with $99.7 \%$ probability 16 hours in advance.

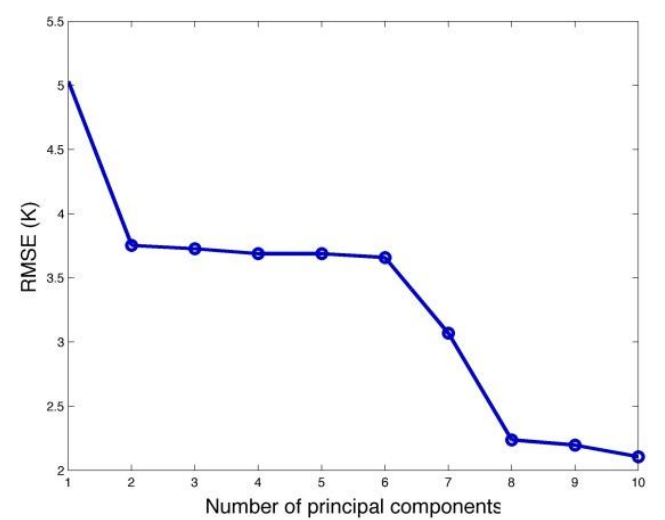

Fig. 3. Results of the K-fold cross validation. Average RMSE as a function of the number of principal components

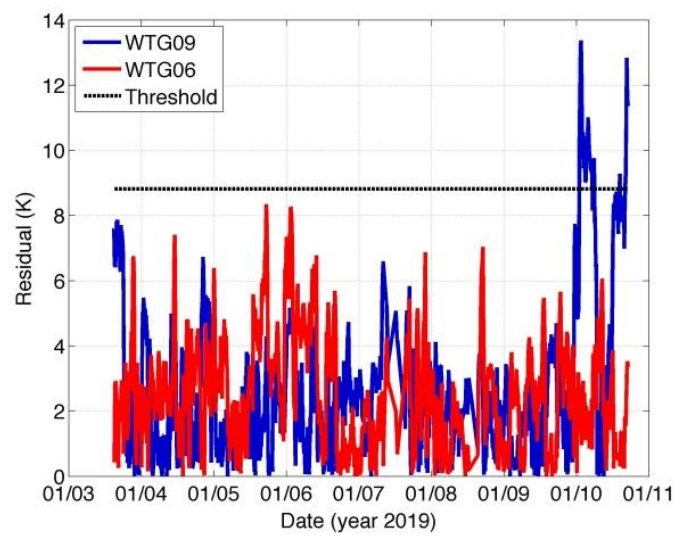

Fig. 4. Time series of absolute difference between generator slip ring temperature measurements and model estimate. Faulty wind turbine (WTG09) and healthy wind turbine (WTG06)

In order to have further quantitative indications, the time series in Figure 4 has been split in two. It is composed of 957 data and the part P1 is selected to include the first 863 data (up to the abnormal increase of the absolute residual for WTG09), the part P2 includes the data from 863 to 957. The statistics for the model testing for parts P1 and P2 are reported in Tables 2 and 3. The mean error
$(M E)$ and the mean absolute error $(M A E)$ are reported. The units are Kelvin degrees.

Table 2: Model testing, WTG06

\begin{tabular}{|c|c|c|}
\hline Data part & $M E$ & $M A E$ \\
\hline P1 & -2.13 & 2.51 \\
\hline P2 & -0.53 & 2.00 \\
\hline
\end{tabular}

Table 3: Model testing, WTG09

\begin{tabular}{|c|c|c|}
\hline Data part & $M E$ & $M A E$ \\
\hline P1 & 0.85 & 2.22 \\
\hline P2 & 6.49 & 6.92 \\
\hline
\end{tabular}

From Tables 2 and 3, it arises that the fault occurrence can be individuated from the remarkable increase of the $M A E$ in period $\mathrm{P} 2$ for the faulty wind turbine WTG09: it becomes of the order of 3.5 times the value for the healthy wind turbine in the same period P2 and 3.5 times the values for the faulty wind turbine in period P1. During period P2, there is a very remarkable increase also of the ME: it is of the order of 7 times of the value recorded during period $\mathrm{P} 1$ for the faulty wind turbine.

The comparison of $M E$ s during P1 for the healthy and the faulty wind turbines indicates that symptoms of a longer-scale evolution of the occurring fault could actually be retrieved. In fact, the same kind of results has been obtained by testing the model on all the data set at disposal (from 1st September 2018 to 11th January 2019): they are not displayed here for brevity. The statistical significance of the long-term evolution analysis of the fault should be addressed in depth [40] and is the object of a further study. From the discussion of the present work, it arises that the fault occurrence can be detected clearly one day before. Since the test case deals with a wind turbine subcomponent that is characterized in general by a quick evolution of the damages, the results of this work are promising and indicate perspectives of future studies about the subject.

\section{CONCLUSIONS AND FURTHER DIRECTIONS}

In the present work, wind turbine fault diagnosis through internal temperature data analysis has been addressed. This topic has been the investigation object of several studies, because the analysis of operation data of wind turbines can in general provide meaningful indication about occurring faults. Previous work from the authors has actually been devoted to the subject $[13,14]$ and was mainly focused on the analysis of wind turbine gearbox temperatures: in that case, the evolution of the fault is in general quite slow (of the order of some months) and therefore operation data analysis could represent a valuable alternative to more powerful and costly condition monitoring techniques (like accelerometers placed at meaningful parts of the gearbox). 
Nevertheless, there are several wind turbine subcomponents (yaw motor, generator bearings and slip ring, and so on) whose monitoring is generally accomplished through temperature sensors. It is therefore important to develop operation and temperature data analysis techniques that could allow timely fault diagnosis at this kind of subcomponents.

On these grounds, in this work a test case of wind turbine generator slip ring fault diagnosis is discussed. The general idea has been modelling the generator slip ring temperature for a healthy wind turbine (from the same wind farm as the faulty one) as a linear function of operation parameters and other internal temperatures (like gearbox temperatures, for example). Since there likely is a remarkable collinearity between some operation variables of wind turbines (as, for example, power rotor revolutions per minute), the decision has been adopting a principal component regression.

The baseline model has been constructed using the data of a healthy wind turbine and has been tested on two data sets: one from a healthy wind turbine and one from the faulty wind turbine. It has been observed that the results do not depend on the particular selection of the healthy test and validation wind turbines and therefore the results can be considered general.

The main result of this study is that it has been possible to identify with clarity the fault occurrence approximately one day before the wind turbine shutdown.

Several are the possible further directions of the present work. The most straightforward development is employing, as is currently being done, the methods and the models of the present work for real-time monitoring of the wind turbines of interest. An important development regards the generalization to other kinds of wind turbines (same technology, different manufacturers), but it should be said that the method is expected to work straightforwardly in the same way.

Another development of this study is including further test cases in the analysis, as for example yaw motor faults, because this kind of damages represents a non-negligible fraction of the unavailability time of wind farms sited in harsh environment, where the flow conditions can be particularly complex [41].

In general, the results of this study should stimulate wind energy practitioners and scholars in adopting complex operation data analysis techniques for condition and performance monitoring.

\section{Acknowledgement}

The authors thank the Lucky Wind company for providing the data sets employed in this work.

\section{REFERENCES}

1 Saenz-Aguirre A, Zulueta, E Fernandez-Gamiz U, Lozano J, Lopez-Guede JM. Artificial neural network based reinforcement learning for wind turbine yaw control. Energies, 2019;12(3): 436. https://doi.org/10.3390/en12030436

2 Astolfi D, Castellani F, Terzi L. Wind turbine power curve upgrades. Energies. 2018; 11(5):1300. https://doi.org/10.3390/en11051300

3 Lee G, Ding Y, Xie L, Genton MG. A kernel plus method for quantifying wind turbine performance upgrades. Wind Energy, 2015;18(7):1207-1219. https://doi.org/10.1002/we.1755

4 Astolfi D, Castellani F, Lombardi A, Terzi L. About the extension of wind turbine power curve in the high wind region. Journal of Solar Energy Engineering. 2019; 141(1): 014501.

https://doi.org/10.1115/1.4041156

5 Petrović V, Bottasso CL. Wind turbine envelope protection control over the full wind speed range. Renewable energy. 2017; 111: 836-848. https://doi.org/10.1016/j.renene.2017.04.021

6 Wang F, Garcia-Sanz M. Wind farm cooperative control for optimal power generation. Wind Engineering. 2018; 42(6): 547-560. https://doi.org/10.1177/0309524X18780377

7 Park J, Law KH. Cooperative wind turbine control for maximizing wind farm power using sequential convex programming. Energy Conversion and Management. 2015; 101: 295-316. https://doi.org/10.1016/j.enconman.2015.05.031

8 Fleming P, Annoni J, Shah JJ, Wang L, Ananthan S, Zhang Z, Chen L. Field test of wake steering at an offshore wind farm. Wind Energy Science. 2017; 2(1), 229-239. https://doi.org/10.5194/wes-2-229-2017

9 Tchakoua P, Wamkeue R, Ouhrouche M, SlaouiHasnaoui F, Tameghe T, Ekemb G. Wind turbine condition monitoring: State-of-the-art review, new trends, and future challenges. Energies. 2014; 7(4): 2595-2630. https://doi.org/10.3390/en7042595

10 Feng Y, Qiu Y, Crabtree CJ, Long H, Tavner PJ. Monitoring wind turbine gearboxes. Wind Energy. 2013; 16(5), 728-740. https://doi.org/10.1002/we.1521

11 Sheng S. Wind turbine gearbox condition monitoring round robin study-vibration analysis (No. NREL/TP5000-54530). National Renewable Energy Lab.(NREL), Golden, CO (United States). 2012. https://doi.org/10.2172/1048981

12 Lin Y, Tu L, Liu H, Li W. Fault analysis of wind turbines in China. Renewable and Sustainable Energy Reviews. 2016; 55: 482-490. https://doi.org/10.1016/j.rser.2015.10.149

13 Astolfi D, Castellani F, Terzi L. Fault prevention and diagnosis through SCADA temperature data analysis of an onshore wind farm. 2014; Diagnostyka, 15.

14 Astolfi D, Scappaticci L, Terzi L. Fault diagnosis of wind turbine gearboxes through temperature and vibration data. International Journal of Renewable Energy Research (IJRER). 2017; 7(2): 965-976.

15 Guo P, Infield D, Yang X. Wind turbine generator condition-monitoring using temperature trend analysis. IEEE Transactions on sustainable energy. 2012; 3(1):124-133. https://doi.org/10.1109/TSTE.2011.2163430

16 Zaher ASAE, McArthur SDJ, Infield DG, Patel Y. Online wind turbine fault detection through automated SCADA data analysis. Wind Energy: An International 
Journal for Progress and Applications in Wind Power Conversion Technology. 2009; 12(6): 574-593. https://doi.org/10.1002/we.319

17 Tautz-Weinert J, Watson SJ. Using SCADA data for wind turbine condition monitoring-a review. IET Renewable Power Generation. 2016; 11(4): 382-394. https://doi.org/10.1049/iet-rpg.2016.0248

18 Dao PB, Staszewski WJ, Barszcz T, Uhl T. Condition monitoring and fault detection in wind turbines based on cointegration analysis of SCADA data. Renewable Energy. 2018; 116: 107-122.

https://doi.org/10.1016/j.renene.2017.06.089

19 Pandit, RK, Infield, D. SCADA-based wind turbine anomaly detection using Gaussian process models for wind turbine condition monitoring purposes. IET Renewable Power Generation. 2018; 12(11): 12491255. https://doi.org/10.1049/iet-rpg.2018.0156

20 Ren Y, Qu F, Liu J, Feng J, Li X. A universal modeling approach for wind turbine condition monitoring based on SCADA data. In 2017 6th Data Driven Control and Learning Systems (DDCLS). IEEE. 2017:265-269. https://doi.org/10.1109/DDCLS.2017.8068080

21 Astolfi D, Castellani F, Terzi L. Mathematical methods for SCADA data mining of onshore wind farms: Performance evaluation and wake analysis. Wind Engineering. 2016; 40(1): 69-85. https://doi.org/10.1177/0309524X15624606

22 Castellani F, Garinei A, Terzi L, Astolfi D, Moretti M, Lombardi A. A new data mining approach for power performance verification of an on-shore wind farm. Diagnostyka. 2013; 14.

23 Marti-Puig, P, Blanco-MA, Cárdenas JJ, Cusidó J, Solé-Casals J. Feature Selection Algorithms for Wind Turbine Failure Prediction. Energies. 2019; 12(3): 453. https://doi.org/10.3390/en12030453

24 Schlechtingen M, Santos IF, Achiche S. Using datamining approaches for wind turbine power curve monitoring: a comparative study. IEEE Transactions on Sustainable Energy. 2013; 4(3): 671-679. https://doi.org/10.1109/TSTE.2013.2241797

25 Schlechtingen M, Santos IF. Wind turbine condition monitoring based on SCADA data using normal behavior models. Part 2: Application examples. Applied Soft Computing. 2014; 14: 447-460. https://doi.org/10.1016/j.asoc.2013.09.016

26 Qiao W, Lu D. A survey on wind turbine condition monitoring and fault diagnosis-Part I: Components and subsystems. IEEE Transactions on Industrial Electronics. 2015; 62(10): 6536-6545. https://doi.org/10.1109/TIE.2015.2422112

27 Cao L, Qian Z, Zareipour H, Wood D, Mollasalehi E, Tian S, Pei Y. Prediction of Remaining Useful Life of Wind Turbine Bearings under Non-Stationary Operating Conditions. Energies. 2018; 11(12): 3318. https://doi.org/10.3390/en11123318

28 Mollasalehi E, Wood D, Sun Q. Indicative fault diagnosis of wind turbine generator bearings using tower sound and vibration. Energies 2017; 10(11): 1853. https://doi.org/10.3390/en10111853

29 Green WH. Econometric analysis. Maxwell Macmillan International. 1997.

30 Astolfi D, Castellani F, Fravolini ML, Cascianelli S, Terzi L. Precision Computation of Wind Turbine Power Upgrades: An Aerodynamic and Control Optimization Test Case. Journal of Energy Resources Technology. 2019; 141(5): 051205. https://doi.org/10.1115/1.4042450
31 Astolfi D, Castellani F, Berno F, Terzi L. Numerical and Experimental Methods for the Assessment of Wind Turbine Control Upgrades. Applied Sciences. 2018; 8(12): 2639. https://doi.org/10.3390/app8122639

32 Astolfi D. A study of the impact of pitch misalignment on wind turbine performance. machines. 2019;7(1): 8 . https://doi.org/10.3390/machines7010008

33 Pozo F, Vidal Y. Wind turbine fault detection through principal component analysis and statistical hypothesis testing. Energies. 2016; 9(1): 3. https://doi.org/10.3390/en9010003

34 Pozo F, Vidal Y, Salgado Ó. Wind turbine condition monitoring strategy through multiway PCA and multivariate inference. Energies. 2018; 11(4): 749. https://doi.org/10.3390/en11040749

35 Pozo F, Vidal Y. Damage and fault detection of structures using principal component analysis and hypothesis testing. In Advances in Principal Component Analysis. 2018:137-191. Springer, Singapore. https://doi.org/10.1007/978-981-10-67044 _ 7

36 Senin N, Moretti M, Leach RK. Shape descriptors and statistical classification on areal topography data for tile inspection in tessellated surfaces. Measurement. 2017;95:82-92.

https://doi.org/10.1016/j.measurement.2016.09.044

37 Basha N, Nounou M, Nounou H. Multivariate fault detection and classification using interval principal component analysis. Journal of computational science. 2018;27:1-9. https://doi.org/10.1016/j.jocs.2018.04.017

38 Jolliffe IT, Cadima J. Principal component analysis: a review and recent developments. Philosophical Transactions of the Royal Society A: Mathematical, Physical and Engineering Sciences. 2016; 374(2065), 20150202. https://doi.org/10.1098/rsta.2015.0202

39 Refaeilzadeh P, Tang L, Liu H. Cross-validation. Encyclopedia of database systems. 2009: 532-538. https://doi.org/10.1007/978-0-387-39940-9_565

40 Zhao Y, Li D, Dong A, Kang D, Lv Q, Shang L. Fault prediction and diagnosis of wind turbine generators using SCADA data. Energies. 2017; 10(8): 1210. https://doi.org/10.3390/en10081210

41 Astolfi D, Castellani F, Terzi L. A study of wind turbine wakes in complex terrain through RANS simulation and SCADA data. Journal of Solar Energy Engineering. 2018; 140(3): 031001. https://doi.org/10.1115/1.4039093

Received 2019-03-03

Accepted 2019-06-10

Available online 2019-06-17

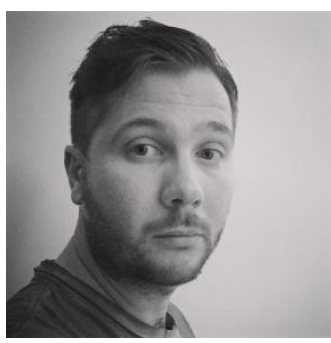

Davide ASTOLFI is a postdoc at the Department of Engineering of University of Perugia, Italy. His research activities deal mainly with modelling and control of mechanical systems, condition monitoring, fault diagnosis, control and optimization of MW-scale wind turbines. He also works on the analysis of the mechanical behaviour of small horizontal axis wind turbines, through wind tunnel testing and numerical modelling. 


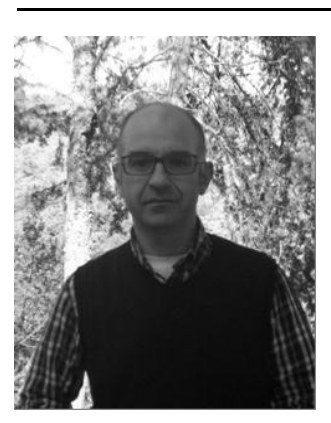

Francesco CASTELLANI is an Associate Professor in Machine Engineering teaching Applied Mechanics at the University of Perugia, Italy. He is involved in many research activities dealing with modelling and control of mechanical systems. His research is focused on numerical and experimental wind turbines studies, wind resource assessment, numerical simulation of wind flow and wakes on complex terrain sites, condition monitoring and fault diagnosis through SCADA data analysis and wind tunnel test of micro wind turbines.

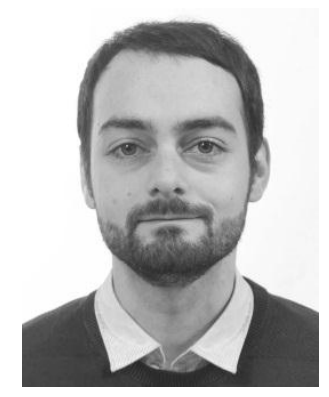

Francesco NATILI is a postgraduate student at the Department of Engineering of University of Perugia, Italy. His research activities deal mainly with modelling and control of wind turbines. 